\title{
La Havane et la Ville de Mexico: une métropolisation par le patrimoine
}

Havana e a Cidade do México: uma metropolização através do patrimônio

La Habana and Mexico City: metropolization through heritage

La Habana y la Ciudad de Mexico: una metropolizacion por medio del patrimonio

\author{
Pierre-M. Le Bel* \\ Felipe De Alba** \\ Luzma Fabiola Nava***
}

Recebido em 20/3/2007; revisado e aprovado em10/9/2007; aceito em 20/12/2007.

\begin{abstract}
Résumé: Les études sur le phénomène de la métropolisation portent surtout sur les très grandes agglomérations urbaines des pays les plus « développés ». Cet article démontre qu'il est aussi possible de voir ce phénomène à l'œuvre dans une ville du « Sud », dans un « régime socialiste » de surcroît. En comparant les stratégies de développement et d'aménagement territorial basées sur des formes de holding des villes de Mexico et de La Havane, nous démontrons comment la réhabilitation patrimoniale joue un rôle dans la stratégie globale de la capitale mexicaine et comment elle est le moyen pour la ville cubaine de participer au réseau des villes mondiales.
\end{abstract}

Mots-clés: La Havane. Patrimoine. Métropolisation.

Resumo: Os estudos sobre o fenômeno de metropolização aplicam-se, sobretudo, às grandes aglomerações do norte. Este artigo demonstra que é também possível ver este fenômeno numa cidade do sul, mesmo sendo num "regime socialista". Comparando as estratégias de reabilitação patrimonial de Havana e da Cidade do México, demonstramos que o patrimônio é o principal meio pelo qual Havana participa na rede das cidades mundiais.

Palavras-chave: Havana. Patrimônio. Metropolização.

Abstract: The studies on the phenomenon of metropolisation relate especially to the very great urban centres of the more "developed" countries. This article shows that it is also possible to see this phenomenon at work in a city of the "South", which is in addition in a "socialist" context. By comparing the strategies of development and territorial management based on business holdings of the City of Mexico and Havana, we show how the patrimonial rehabilitation plays a part in the wider strategy of the Mexican capital and how it is the main way for the cuban city to participate to the network of world cities.

Key words: Havana. Patrimony. Metropolization.

Resumen: Los estudios sobre el fenómeno de la metropolización se focalizan sobre todo en las grandes ciudades de países del Norte. Este artículo demuestra que es posible pensar este fenómeno en una ciudad del "Sur", en un régimen autollamado socialista. Comparando las estrategias de desarrollo y planificación territorial basadas sobre la idea de "centros de negocios" de la ciudad de México y la Habana, demostramos cómo la rehabilitación patrimonial juega un rol en la estrategia global de la capital mexicana y como elle es el medio para la ciudad cubana de integrarse a la red de ciudades mundiales.

Palabras clave: La Habana. Patrimonio. Metropolización.

\section{Introduction $^{1}$}

À la fois cause et effet de la mondialisation, le phénomène de la métropolisation à l'échelle planétaire constitue un processus de grande importance pour les sociétés contemporaines (DE ALBA et JOUVE, 2005). La métropolisation est liée au développement des nouvelles technologies de l'information et des transports qui a permis de re-territorialiser les pratiques quotidiennes des habitants des plus grandes agglomérations urbaines, des entreprises et des gouvernements. Le quartier perd ainsi du terrain en matière d'unité de base de la quotidienneté en faveur de la région métropolitaine (ASHER, 1998). On assiste à une dilatation de la zone métropolitaine, à une fragmentation des zones de bâti et au développement d'agglomérations urbaines polycentriques. Dans ces centres multipliés au sein d'une même ville sont concentrées les populations les plus qualifiées. Ces phénomènes s'accompagnent d'une polarisation commerciale de la zone métropolisée. Les entreprises sont attirées par les centres parce qu'il est plus facile pour elles $d^{\prime} y$ trouver des gens qualifiés et des

\footnotetext{
*Univ. d'Ottawa. plebe031@uottawa.ca.

** Univ. de Montreal. fdj.de.alba.murrieta@umontreal.ca.

*** Univ. du Québec à Montréal. nava jimenez.luzma fabiola@courrier.uqam.ca
} 
fournisseurs de services - sous-traitants (ASHER, 1995; VELTZ, 2000; SASSEN, 1996; 2002). Toute cette dynamique est d'abord possible grâce à la mise en réseau des villes. $C^{\prime}$ est le réseautage qui permet la reterritorialisation. Selon nous, la métropolisation est donc d'abord, au sens le plus fondamental, le phénomène par lequel une ville participe au réseau des villes. Cette participation s'inscrit plus à l'intérieur d'un continuum que par sa présence ou son absence. Une des conditions premières à l'atteinte du statut de ville métropolisée est son intégration aux flux globaux qui à la fois structurent et favorisent le capitalisme avancé - finances, activités économiques d'intermédiation, recherche et développement, etc. ${ }^{2}$ (PIERRE et PETERS, 2000). Par la métropolisation, on assiste à une nouvelle polarisation du système mondial qui est de moins en moins régi par l'État, qui agissait auparavant comme le principal espace de régulation économique (TAYLOR, 1995).

Cette modification de nos références géopolitiques et l'éclosion des métropoles globales avaient fait l'objet d'importantes discussions principalement au sujet des villes de pays dits développés (SASSEN, 1996; DE ALBA et JOUVE, 2006). Jusqu'à il y a quelques années, l'insertion des villes des pays en développement dans les flux globaux de la nouvelle économie a été sous-estimée et son étude négligée. Nous constatons un oubli similaire des villes de pays qui ont été pendant des décennies sous l'égide de gouvernements d'orientations dites socialistes. La situation a aujourd'hui changé. Il s'est produit une libéralisation des villes de « $\mathrm{l}^{\prime}$ Est » et une intégration des villes du «Sud» au phénomène connu comme mondialisation, processus qui a suscité un débat sur les relations d'interdépendance qui liaient ces villes, ou métropoles selon le cas, avec leur fondement qu'on croyait jusqu'à il n'y a pas si longtemps naturel : le territoire national (BELLEY, 2002 ; DE ALBA et JOUVE, 2006).

Dans cet article, nous analysons le cas de la ville de La Havane et montrons comment elle peut prétendre participer au réseau des villes métropolisées, pourvu que dans la mesure où l'on veut bien centrer l'analyse sur autres choses que des flux globaux d'investissements ou des recherches en biotechnologie. Nous avons recours, pour ce faire, à une explication du processus de réhabilitation patrimoniale à La Havane et faisons une comparaison rapide et inédite entre la stratégie havanaise et celle de la ville de Mexico en la matière.

\section{La Havane Métropolisée? ${ }^{3}$}

À première vue, il peut sembler surprenant de parler de métropolisation pour qualifier le processus auquel participe la capitale cubaine. La Havane n'a pas l'envergure démographique de Mexico, ni sa puissance économique. Pourtant, la ville de deux millions d'habitants participe bel et bien au réseau des villes mondiales. Il importe cependant de bien faire ressortir les modalités de cette participation puisqu'on ne parlera évidemment pas dans ce cas de fonctions de commandes financières mondiales.

Sur le plan national, il ne fait aucun doute que l'agglomération urbaine constituée par les 15 municipalités rassemblées dans la province de La Havane constitue l'ensemble métropolitain le plus important du pays. Si Santiago fut d'abord le centre administratif et culturel de l'île, la localisation stratégique de La Havane en fit rapidement un site d'importance. Déjà en 1950, la primauté de la Havane à Cuba est indiscutable et celle-ci se compare aux autres métropoles latinoaméricaines. À cette époque, La Havane est six fois plus grosse que Santiago de Cuba. Plus d'importations et d'exportations transitent par son port, plus de gens y vivent et y migrent, elle participe davantage au PIB et concentre plus d'emplois (SCARPACCI et al. 2002). Le poids démographique de la métropole cubaine dans son territoire national, $21 \%$ en 1953, est comparable avec ceux de Buenos Aires $(29,1 \%)$ ou Santiago de Chile $(25,4 \%)$ alors que Mexico ne concentre à cette époque que $12,2 \%$ de la population nationale et que Lima n'en a que 10,4\% (UNU, 2004). La Havane est à cette époque un centre culturel comparable à Mexico ou Buenos Aires. On y produit des radio-romans qui sont diffusés partout en Amérique latine, on exporte sa musique jusque dans les cabarets de New York. Déjà, la Vieille Havane est un lieu de rencontre pour les habitants et d'exploration pour les 
touristes. Comme bien des capitales latinoaméricaines, La Havane a bénéficié des gouvernements fortement centralisés au détriment de la campagne.

La révolution a rapidement changé les choses. Le développement des capitales provinciales et des campagnes a pris le pas sur celui de la capitale nationale. Même si La Havane est restée le cœur économique du pays et son agglomération de loin la plus populeuse, son développement urbanistique et social fut pour le moins mitigé, notamment dans la municipalité de La Vieille Havane. Cette dernière devint un des lieux les plus socialement défavorisés de l'île, la mortalité infantile y était plus haute que partout ailleurs dans l'agglomération, ses infrastructures étaient fortement déficientes, voire dangereuse dans le cas du bâti résidentiel. En 1991, la fin du bloc soviétique, les pressions économiques vécues au niveau national se sont ajoutées à la détérioration de la municipalité. Le gouvernement cubain prit alors la décision d'axer ses efforts de développement autour du tourisme. La Vieille Havane avait non seulement besoin d'intervention urgente, elle était également un lieu patrimonial à la valeur reconnue puisqu'elle était entrée au patrimoine mondial de 1'UNESCO en 1982. En restaurant le quartier, l'État attirerait des capitaux et la municipalité sortirait de son marasme.

En 1993, le Conseil d'État donne au Bureau de 1'Historien de la Ville de La Havane $^{4}$ le pouvoir de créer ses propres entreprises, de lever des impôts sur les entreprises qui tirent leur profit de son territoire et de conclure des accords avec des partenaires étrangers. Cet acteur aux pouvoirs considérablement augmentés prend alors place dans un espace autrefois occupé par d'autres instances politiques. D'une part, non seulement le gouvernement provincial qui est habituellement maître dans le secteur touristique perdait son pouvoir sur la capitale en ce domaine, mais il fut aussi bien vite devant un partenaire qui avait des moyens financiers plus importants que les siens (puisque, entre autres, le Bureau faisait affaires en dollars US et non en pesos cubains). D'autre part, la municipalité perdait le pouvoir effectif sur le construit à haute valeur patrimoniale, soit plusieurs centaines de bâtiments et des places publiques. Enfin le ministère du tourisme, normalement seul habilité à établir des joint ventures dans ce secteur, avait dorénavant un compétiteur. Aujourd'hui, toutes ces instances sont réunies en un Grupo de control, une structure de concertation qui dirige le développement territorial de la Vieille Havane. À cette structure sont étroitement associés les Conseils Populaires (CP), des instances locales créées en 1976 qui sont composées de délégués municipaux et provinciaux, de membres d'organisations de masse ou de simples citoyens. Les CP servent « au contrôle des services et à la mobilisation des ressources dans des aires géographiques spécifiques » (DILLA et OXHORN, 2002). Les Conseils populaires semblent en être venus à tenir le rôle d'intermédiaires entre le Bureau de l'Historien, la municipalité et les citoyens.

L'Historien de la ville de la Havane, c'est aussi un individu en chair et en os. Il s'agit d'Eusebio Leal Spengler, entre autres connu pour avoir assumé publiquement sa confession catholique alors qu'il est délégué à l'Assemblée nationale. Il possède une renommée mondiale, mise à profit dans la restauration d'autres centres historiques comme celui de Mexico mais aussi de Quito, en Équateur. Ami personnel de Fidel Castro, la forte personnalité de l'historien et son projet de réhabilitation attirent les appuis de l'étranger autant qu'à Cuba.

Le travail accompli par le Bureau de 1'Historien depuis plus de 13 ans est exceptionnel. Les profits tirés du tourisme et des impôts sont redistribués de la façon suivante : $45 \%$ sont réinvestis dans le patrimoine et les infrastructures touristiques, $35 \%$ sont destinés aux œuvres sociales et $20 \%$ sont versés au gouvernement central. Les changements sont perceptibles ailleurs que dans le domaine architectural, en lui-même spectaculaire. Des écoles, cliniques, sales sportives, programmes de sensibilisation en santé et en patrimoine ont été mis en place. La population de la municipalité est passée de 71000 en 1995 à 66000 en 2001 (OFICINA NACIONAL de ESTADISTICAS, 2002), ce qui reflète le travail du Bureau en matière de tertiarisation, essentielle à la stimulation économique et à la sécurité des infrastructures. Les conditions sanitaires des logements 
s'améliorent également. Le Bureau de l'Historien, avec ses 10000 employés, est l'employeur le plus important de la municipalité.

La réhabilitation patrimoniale, stimulée par cet acteur puissant, est également le cadre de nouveaux et nombreux liens avec des acteurs étrangers. D'abord $l^{\prime}$ UNESCO, qui dans un premier temps a attribué le label de Patrimoine de l'Humanité au centre historique, mais aussi l'Organisation des villes du patrimoine mondial. Le PNUD est l'instance onusienne la plus impliquée. Il gère la coopération décentralisée qui n'implique aucun gouvernements centraux mais plutôt des ONG, des universités ou des municipalités, surtout européennes. Plusieurs dizaines d'ONG et d'organismes gouvernementaux de développement font aussi affaire à la Vieille Havane indépendamment du PNUD.

La façon de faire le développement social en passant par le secteur touristique a pour conséquence qu'il faut d'abord restaurer les zones à haute valeur patrimoniale afin d'attirer les touristes et leurs dollars. En outre, l'acteur le plus puissant économiquement est le Bureau de l'Historien dont les pouvoirs ne s'appliquent qu'aux édifices patrimoniaux. Les ONG, entreprises étrangères, et gouvernements ont plus tendance à investir sur son territoire parce que ses pouvoirs économique et politique donnent plus de chances de réussite à leurs projets. Ainsi les secteurs les moins pourvus en patrimoine bénéficient le moins de la stratégie et ce, peu importe les divisions administratives. Ne peuton pas y voir l'ébauche d'un morcellement de l'espace urbain calqué sur le modèle des villes métropolisées?

La coopération décentralisée entre des localités européennes et le centre historique participe à l'affaiblissement du rôle de l'État dans le développement local. Cet affaiblissement existe à Cuba comme dans le monde capitaliste et, comme ailleurs, il n'est pas également réparti. Il existe d'autres Bureaux de l'Historien ailleurs à Cuba qui n'ont pas des pouvoirs aussi importants. À Cuba, La Havane est la seule agglomération urbaine pesant suffisamment lourd pour prétendre à un rôle métropolitain. $C^{\prime}$ est non seulement là que passe la majeure partie du tourisme, mais c'est aussi dans la capitale que résident la plupart des citoyens ayant de la famille à l'étranger, qui peuvent donc participer au réseau des remesas (envois d'argent par la diaspora cubaine). Cette décentralisation devient plus révélatrice si l'on prend en considération que le tourisme, le patrimoine et l'aide internationale sont parmi les rares secteurs pour lesquels l'île des Caraïbes possède des relations avec l'étranger, particulièrement avec les pays du « Nord ». Même des échanges commerciaux ténus ne signifient pas une indépendance face à la mondialisation de l'économie. Le recul momentané du tourisme après le 11 septembre 2001, la fluctuation des travaux suivant les mesures humanitaires de la Communauté européenne tout comme le développement économique et social de la Vieille Havane sont des conséquences de la connectivité de cette dernière au reste du monde.

\section{À la Havane comme à Mexico?}

Dans le but d'élargir la réflexion sans prétendre l'épuiser, arrêtons-nous maintenant au cas du centre historique de la ville de Mexico. La capitale du Mexique participe pleinement au processus de mondialisation, processus qui conduit à une accélération et à une intensification de ses échanges avec certaines villes entre lesquelles il existe une hiérarchie claire (SASSEN, 2002). Quatrième mégalopole du monde, avec 22 millions d'habitants, elle se présente comme un cas de " macrocéphalie urbaine » typique des pays latino-américains (SEMMOUD, 2001). Cette métropole dispose de plus du $20 \%$ de la population nationale et contribue à 30\% du PIB national mexicain (DE ALBA, 2005). Tant par les ressources concentrées sur son territoire que par les dynamiques sociales et politiques qui y ont lieu, la métropole de Mexico a été le pôle stratégique du régime politique mexicain. Par ailleurs, la ville de Mexico a été le principal laboratoire de transformation de l'État mexicain durant les vingt dernières années. Cette période s'est caractérisée par l'application d'un agenda néo-libéral. Elle a aussi été le cadre de la manifestation de nouveaux acteurs politiques et sociaux qui ont substantiellement modifié la scène 
politique nationale et locale. Mexico est à la fois le creuset et le laboratoire de ces transformations (DE ALBA, 2005).

La ville de Mexico et sa zone métropolitaine sont un exemple de l'explosion démographique qu'ont connue bon nombre de métropoles de l'Amérique latine depuis cinquante ans (RODRÍGUEZ et al. 1997; STREN, 2000; DE ALBA, 2005). Diverses variables historiques et structurelles ont été combinées pour conduire à cette dynamique comme l'arrivée massive d'une population rurale et indigène attirée par l'image du progrès et de justice sociale que le gouvernement fédéral a donnée à la ville (DAVIS, 1998). En effet, une des particularités du régime politique résidait dans le lien organique entre l'Etat fédéral, contrôlé par un parti politique dominant (le PRI) et la création, en 1928, du District fédéral. Jusqu'aux années soixante-dix, c'est Mexico qui a le plus profité des investissements massifs provenant de l'État fédéral (ICAZURIAGA 1992). Ces investissements ont permis à ses habitants de tirer bénéfice d'un niveau de vie stable et prospère, ainsi que d'une relative mobilité sociale. Progressivement, les investissements industriels de l'État mexicain ont été réorientés vers le nord-ouest de la métropole, dépassant les limites de la ville. Au même moment, les délégations du District fédéral et des communes de l'est et du sud-est de la métropole ont reçu l'essentiel de l'exode rural provenant $d u$ reste du pays. À partir des années soixante-dix, l'investissement politique industriel de l'État mexicain destiné à la capitale a diminué alors que sa population augmentait fortement. Ceci a conduit à une forte polarisation sociospatiale qui aura des conséquences très importantes dans l'organisation politique de la métropole. Si les décennies 1960-1970 ont été marquées par une explosion démographique à l'échelle de la métropole, les années 1980 se caractérisent par une diminution relative $\mathrm{du}$ poids démographique du District fédéral par rapport à la croissance des communes périphériques (FAUNDEZ et al. 1997). La crise économique de 1982 et le séisme dévastateur qui toucha la ville de Mexico en 1985 ont impulsé un renforcement des groupes communautaires et un virage néolibéral qui a affaibli le gouvernement central.
Le centre historique de la ville de Mexico (CHVM) a connu, au rythme de la croissance métropolitaine, de multiples phases, évolutions et bouleversements. Le CHVM, qui pendant des siècles avait représenté le pilier politique, économique et religieux du pays, a souffert d'une constante détérioration. La dévastation est arrivée à un point tel qu'un tiers de sa population l'a abandonné (OSAVA, 2005). Aujourd'hui, le CHVM comme la Vieille Havane partagent des problèmes propres aux grandes villes : détérioration des bâtiments historiques, marginalité des habitants, délinquance, croissance des réseaux de commerce informel de même que le manque de ressources budgétaires pour alléger de tels maux.

C'est dans le contexte d'une rencontre entre les gouvernements fédéral et municipal et d'acteurs privés que fut créé en 2001 le Conseil consultatif du Centre historique. Quelques personnes ont pris part à sa formation, dont l'entrepreneur Carlos Slim, considéré comme l'homme le plus riche de l'Amérique latine et président du Conseil. Selon lui, le Conseil consultatif a comme objectifs la réhabilitation du centre historique, la diminution de l'extraction excessive de l'eau, l'amélioration du niveau de vie des habitants de cette zone et la stimulation de l'économie (DE ALBA, 2005). À l'époque, la création de l'organisme a été vue comme une occasion d'attirer l'investissement privé dans le centre historique de la ville. La présence d'une telle personnalité du monde de la finance légitime en partie les actions du Conseil. Les deux paliers de gouvernement municipaux et fédéraux ont tous deux injecté des sommes totalisant près de $100 \mathrm{M}$ US, en partie sous forme d'incitatifs fiscaux (GRAJEDA et RUIZ, 2003).

Le gouvernement mexicain a également créé en 2003, la Société anonyme Centre historique qui, avec un capital initial qui était de 90,6 millions de dollars sur une période de 2 ans, possédait déjà 66 monuments historiques. Cette société a été formée par trois grands actionnaires : Immobilière Carso, Groupe Financier Inbursa et la Controladora de Servicios de Telecomunicaciones. La Société est entrée à la Bourse mexicaine des valeurs en 2003, ce qui illustre son orientation néolibérale. La fondation Telmex, propriété 
de Carlos Slim, injecte massivement des fonds vers le développement social par l'intermédiaire de la Société anonyme Centre historique en mettant l'accent surtout sur l'assistance médicale, la promotion de l'emploi et l'appui aux handicapés. 10000 emplois directs auraient été créés par la réhabilitation du centre historique. De plus, le projet dépasse ce qui pourrait être considéré comme une série de mesures à caractère local puisque le Slim est également l'instigateur du Promoteur pour le développement économique de l'Amérique latine (IDEAL), dont l'objectif est de financer des projets d'infrastructure ailleurs au sud du Rio Grande. Au-delà de cette vision conquérante de la réhabilitation patrimoniale, le Mexique reste un pays pionnier et clé dans le processus de récupération de monuments historiques en Amérique latine ${ }^{5}$. Une si grande activité de restaurations de monuments historiques a exigé l'assistance de spécialistes nationaux et internationaux, comme l'Historien de la ville de La Havane, Eusebio Leal Splenger. .

Mexico et La Havane ont leurs différences, soit, mais aussi de nombreux éléments comparables. Les deux sont des villes primatiales chez elles et sont aux prises avec les problèmes liés à cette primauté. Chacune a un centre historique important faisant partie du patrimoine mondial de l'UNESCO. Toutes deux y ont fait leur entrée quelques temps avant d'entreprendre leurs grands projets de restauration. Le label de patrimoine mondial a joué un rôle important dans les stratégies de marketing urbain des deux agglomérations et cela a fortement contribué à leur arrimage au réseau des villes mondiales. Les régimes politiques sont évidemment aux antipodes et les dimensions des villes, des nations sont sans commune mesure, mais ce que nous avons essayé de démontrer, c'est que les points communs sont suffisamment nombreux pour poursuivre une discussion sur leurs façons de participer à la métropolisation. Nous avons porté notre attention sur leurs manières d'intégrer la réhabilitation patrimoniale à leur stratégie métropolitaine et leur façon de la légitimer.

Les deux projets de réhabilitation de centre historique s'articulent autour de groupes d'acteurs où un personnage et son institution, non-étatique mais avec le souti- en presque inconditionnel de l'État, occupe la place de leader. À Mexico, Carlos Slim et la Sociedad del Centro Historico de la Ciudad de Mexico S.A. de C.V. ont assis leur légitimité sur la sécurité financière et budgétaire qu'impliquait leur participation aux yeux de l'État et de la population. La variété et la vigueur des entreprises sous la bannière de Slim constituaient une assurance que le projet aboutirait à une réussite à la fois sociale, patrimoniale et financière. En amont du projet, ici comme à Cuba, des demandes sociales en matière de logement, d'emplois et de distribution d'eau, pour ne mentionner que celles-là, étaient mises de l'avant par les citoyens et furent reprises par les entités leader afin de légitimer leurs projets et, du coup, offrir une solution à des problèmes jusqu'alors aux mains de l'État.

À La Havane, Eusebio Leal Spengler et le Bureau de l'Historien de la Ville de La Havane légitiment leurs actions auprès de la population par la personnalité même de l'Historien, ses compétences patrimoniales et culturelles. Dans la hiérarchie politique, son leadership est facilité par ses rapports avec le Conseil d'État. Le Bureau dans son ensemble tire également une légitimité de la coopération internationale en matière de développement socio-économique qu'il réussit à attirer et de la participation d'organismes internationaux qui oeuvrent dans le patrimoine. Cette stratégie sort du cadre politique imposé à Cuba mais est facilitée par le volet social et identitaire de la réhabilitation patrimoniale.

Comme dans bien des cas de réhabilitation de centre historique, celui de Mexico a donné cours à un phénomène de gentrification qui est loin d'être aussi évident dans le cas de la Vieille Havane. Par contre, les habitants du centre historique de cette dernière $y$ ont plus d'opportunités économiques puisque c'est là surtout que les touristes y laissent leurs dollars, le Bureau de l'Historien ses investissements en infrastructures et les organismes internationaux une bonne part de leur aide.

Le succès des deux entités leader de la restauration fait qu'elles ont pu profiter de leur avantage sur le gouvernement pour s'approprier un territoire, un champ d'action toujours plus grand. Carlos Slim souhaite 
étendre les actions de son entreprise à des projets dans toute 1'Amérique latine par l'entremise de la Impulsora para el desarrollo economico en América Latina (IDEAL). Les deux ont recours à l'œil bienveillant de l'échelle internationale afin de légitimer leurs projets. Mexico s'en est servi pour faire voir que le Mexique était prêt à laisser une bonne marge de manœuvre au secteur privé, et c'est aussi un peu le cas en ce qui concerne les entreprises touristiques étrangères. La Sociedad del centro historico de la ciudad de Mexico S.A. de C.V. est coté en bourse, ce qui montre bien son arrimage au libre marché et la rentabilité d'un projet patrimonial. De plus, il semble que Slim mette, à côté de ses valeurs mercantilistes, une idéologie tournée vers l'Amérique latine dans le but de faire contrepoids à l'hégémonie nordaméricaine. On peut voir dans la collaboration entre Eusebio Leal et Carlos Slim la preuve de la construction graduelle de liens entre deux régimes complètement différents mais entièrement justifiés par leur contexte respectif. Dans le cas de La Havane, ces liens et l'entreprise patrimoniale ont une importance stratégique plus grande pour l'agglomération et la nation cubaine que dans le cas mexicain où elle s'insère dans une stratégie à plusieurs volets dans laquelle le patrimoine ne joue pas le même rôle d'avant-plan. Mais il aura peutêtre un effet international de plus en plus important, comme ce fut le cas à La Havane, parce qu'il est porteur d'un sentiment identitaire fort et instrumentalisé par l'acteur économique principal.

En somme, il s'agit là de deux cas qui défient la comparaison sans que celle-ci ne soit impossible, loin de là. Cela démontre que la réflexion sur la métropolisation mérite d'être alimentée à partir des villes du « Sud » (DE ALBA et JOUVE, 2006). Parmi celles-ci, La Havane a choisi la voie patrimoniale et touristique comme vecteur de métropolisation, et Mexico l'a intégrée à sa stratégie globale. Nous avons vu que le patrimoine peut jouer un rôle significatif dans la légitimation des processus, des impacts économiques, politiques et sociaux de la métropolisation.

Il ne faut pas croire qu'il faille établir une relation de nécessité entre la métropolisation et l'économie de marché. Le régime politique et économique ne détermine pas le fait qu'il y ait ou pas métropolisation. Il existe des stratégies alternatives pour s'intégrer au réseau des villes sur l'échiquier mondial. Nous croyons avoir démontré par nos exemples que c'est dans les structures unissant les différents acteurs et dans l'imbrication de leurs actions portées sur de multiples échelles qu'on peut trouver les conditions de la participation au réseau des villes globales (DE ALBA, 2005).

\section{Notes:}

${ }^{1}$ Nous voulons remercier Paulina Gomez Luévano (University College London, UK) pour la lecture d'une version initiale de ce texte.

${ }^{2}$ Pour des discussions sur les multiples définitions possibles du concept, voir LACOUR et PUISSANT, 1999; également BASSAND, 1997.

${ }^{3}$ Les données sur le processus de réhabilitation de la Vieille Havane ont été rassemblées à La Havane au cours de 31 entretiens semi-dirigés auprès de multiples acteurs entre septembre 2003 et décembre 2003, d'une recherche documentaire approfondie ainsi que de 4 entretiens semi-dirigés menés à La Havane en juillet 2006. Voir aussi LE BEL, 2005.

${ }^{4}$ Cette instance existait depuis 1938 avec des pouvoirs essentiellement consultatifs.

${ }^{5}$ Le pays compte actuellement 25 emplacements inscrits dans la liste du Patrimoine Mondial de l'UNESCO, dont 22 sont de caractère culturel, le reste étant de caractère naturel.

\section{Références}

ASCHER, François. Métapolis ou l'avenir des villes. Paris: Odile Jacob, 1995.

La fin des quartiers? In: Nicole Haumont (dir.) L'urbain dans tous ses États. Faire vivre, dire la ville. Paris et Montréal: L'Harmattan, 1998. p.183-201.

BASSAND, Michel. Métropolisation et inégalités sociales. Lausanne: Presses polytechniques et universitaires romandes. 1997.

BELLEY, S. Des communautés urbaines aux communautés métropolitaines: quelles innovations institutionnelles? In: Organisations et territoires 11, p.51-57, 2002.

BORJA, J.; DE ALBA Felipe; IRACHETA, A.X. El desafío metropolitano. Mexico-D.F.: PUEC-UNAM, 2004.

DAVIS, D.E. El Leviatán Urbano. La Ciudad de México en el siglo XX. México: FCE, 1998.

DE ALBA, Felipe. Geopolítica metropolitana del Valle de México: crisis o reconfiguración institucional? In: El desafío metropolitano. BORJA, Jordi; WARIO, H. Esteban; DE ALBA, Felipe; IRACHETA, Alfonso X. México: PUEC-UNAM-ALDF, 2004. p. 121-57.

Geopolítica metropolitana, el manejo del conflicto y la "crisis permanente" en el entorno de cambios políticos de la metrópolis de México. Revista Anuarios Espacios Urbanos, México, UAM, p. 57-78, junio/septiembre 2005. 
La hidropolítica en la crisis decisional de la metrópoli de México: Coyuntura o síntomas de colapso?. Revista Ciudades, no. 66.2005

- ¿Es manejable la megalópolis de México? Cuando la modernidad se transforma en disputa clientelar. Análisis de la 'crisis de Texcoco'. En revista Territorios Numero 12: 31-52. 2005.

Geopolítica metropolitana y gobernabilidad del conflicto en la metrópolis de México: seis hipótesis para discutirla. Territorios, n. 14: 119-42, 2005.

DE ALBA, Felipe; NAVA, Luzma Fabiola; NOISEUX, Yanick. "Neoliberalism and Water Privatization in Mexico City, Institutional and Economic Actors, One Decade After the Reforms".

DE ALBA, F.; HERNANDEZ, S.; GONZALEZ, M.; RAMOS, R.. Agenda Metropolitana, GDF, CGPDyM, 1999.

DE ALBA, Felipe; JOUVE, Bernard. Mexico entre flux globaux, trransformations politiques et recherche d'une «troisième voie ». In : DES FLUX ET DES TERRITOIRES, Montréal: Presses de l'Université du Québec, 2006. p.47-70.

DE ALBA, F.; KLOSTER, K. Politic Fragmentation and Social Conflicts Through the Water Divergence on the Metropolis of Mexico, 2005

DILLA, H.; OXHORN, P. The Virtues and Misfortunes of Civil Society in Cuba. In: Latin American Perspectives, 29(4), p.11-30, 2002.

FAUNDEZ, M.A. et al. Modernización institucional de las politicas sociales en América Latina: el caso de Chile, Brasil, Argentina y México. Mexico: Proyecto MostFLACSO, 1997.

GRAJEDA, Ella; RUIZ, José Luis. Invierte la IP 4 mil mdp en rescate de Centro Histórico In: El universal, 30 / 05/2003 http://www2.eluniversal.com.mx/

ICAZURIAGA, C. La metropolización de la ciudad de México a través de la instalación industrial. Tlalpan-México: Ediciones de la Casa Chata, CIESAS, 1992.

JOUVE, Bernard; ROCHE, Yann. Des flux et des territoires: vers un monde sans États? Montréal: Presses de l'Université du Québec, 2006.

JOUVE, Bernard, LEFÈVRE, Christian. Villes, métropoles: les nouveaux territoires du politique. Paris: Anthropos, 1999.

LACOURT, Claude; PUISSANT, Sylvette. La Métropolisation: Croissance, Diversité, Fractures. Paris: Anthropos, 1999.

LE BEL, Pierre-Mathieu. La réhabilitation de la Vieille Havane: le local dans le global en milieu socialiste. Montréal: CRISES, 2005.

MONNET, Jérôme. Mexique, Amérique latine et Amériques face à la métropolisation et à la mondialisation. Réflexions sur la permanence des cadres de l'analyse géographique. In: Historiens et géographes, 374, mai, p.369-378, 2001.

NONJON, Alain; DALLENNE, Pierre. La mondialisation: genèse, acteurs et enjeux. Paris: Ellipses, 2004.

Officina del Historiador de la Ciudad de La Habana. San Isidro, la nueva imagen. La Havane: Éditions Boloña, 1998a.
Plan de desarollo integral. Avance. La Havane: $\mathrm{OHCH}, 1998 \mathrm{~b}$.

Boloña, 2001.

Desafío de una utopía. La Havane : Éditions

Oficina del Historiador de la Ciudad de La Habana et al. (Censo de población y viviendas, Centro Historico, La Habana Vieja 1995. La Havane : OHCH, 1995a.

Censo de población y viviendas, Centro Historico, La Habana Vieja 1995. Tablas preliminares seleccionadas. La Havane : OHCH, 1995b.

Oficina Nacional de Estadisticas. Anuarios Estadistico de Cuba 2001. La Havane : ONE, 2002.

ORMINDO, P. Formacion en Conservacion de monumentos y gestion integral del Patrimonio en América Latina y el Caribe. In : Proyecto Gestion Integral del Patrimonio Cultural, Oficina Regional de Cultura para la América Latina y el Caribe, UNESCO, p.13-40, 2003.

OSAVA, M. Megaciudades se vacían. Metrópolis brasileñas como Río de Janeiro y Sao Paulo pierden densidad poblacional y calidad de vida. Mexico: IPS, No2, 2005.

PREVOT SCHAPIRA, Marie-France (coord.). Dossier : métropoles d'Amérique latine, de l'espace public aux espaces privés. In : Cahiers des Amériques latines, 35, p. 15-137, 2001.

RODRIGUEZ ALOMA, Patricia. El centro Historico de La Habana : un modelo de gestion publica. In : FLACSO (éd.) Centros Historicos de América Latina, Quito: Editorial FLACSO, p.217-236, 2002.

Un Nuevo Enfoque para el Manejo de Areas Antiguas, In : UNESCO (éd.) Proyecto Gestion Integral del Patrimonio Cultural, Oficina Regional de Cultura para la América Latina y el Caribe, UNESCO, p.41-73, 2003.

RODRÍGUEZ, A, et L.WINCHESTER. (coord.) Ciudades y gobernabilidad en America Latina. Santiago de Chile: SUR, 1997.

SASSEN, Saskia. La ville globale. New York, Londres, Tokyo. Paris: Descartes, 1996. 2002.

Global networks, linked cities. New York, Routledge,

SCARPACI, Joseph L. et al. Havana : Two Faces of the Antillean Metropolis. Chapel Hill: University of North Carolina Press, 2002.

SEMMOUD, B. Introduction à la géographie des très grandes villes. Paris : Éditions du Temps, 2001.

STREN, R.E. New Approaches to Urban Governance in Latin America. Toronto: Centre for Urban and Community Studies, University of Toronto, 2000.

TAYLOR, PJ. World cities and territorial states: the rise and fall of their mutuality. In : P.L. Knox et P.J. Taylor (éds.) World Cities in a World-System, Cambridge: Cambridge University Press, p.48-62, 1995.

UNU (Universidad de las naciones unidas), 2006, http:/ /whc.unesco.org/

VELTZ, Pierre. Le développement local face à la mondialisation. In : A. Ménéménis (éd.) Comment améliorer la performance économique des territoires? Paris : Société des acteurs publics, 2000. 